\title{
The Huggable Robot Probo, a Multi-disciplinary Research Platform
}

\author{
Kristof Goris ${ }^{\star}$, Jelle Saldien, Innes Vanderniepen, and Dirk Lefeber \\ Vrije Universiteit Brussel, Robotics \& Multibody Mechanics Research Group, \\ Pleinlaan 2, 1050 Brussel, Belgium \\ \{kristof.goris, jelle.saldien, innes.vanderniepen, dirk.lefeber\} @ \\ vub.ac.be \\ http://anty.vub.ac.be
}

\begin{abstract}
The concept of the huggable robot Probo is a result of the desire to improve the living conditions of children in hospital environment. These children need distraction and lots of information. In this paper we present the concept of this new robot. The robot will be employed in hospitals, as a tele-interface for entertainment, communication and medical assistance. To communicate according to social rules, the robot needs the ability to show facial expressions. Using a well defined set of Action Units (AU) it's possible to express some basic emotions. A prototype of the robot's head, capable of showing these basic emotions is presented. In order to express emotions, an emotional interface is developed. The emotions, represented as a vector in an 2D emotion space, are mapped to the DOF used in the robot. A graphical user interface to control the virtual and real prototype is also presented.
\end{abstract}

Key words: emotional interface, human-robot interaction, huggable robot, multidisciplinary research platform

\section{Introduction}

A hospitalization can have serious physical and mental influences, especially on children. It confronts them with situations that are completely different from the these at home. In hospital, a child's experiences are more limited due to the closed and protective environment, which leads to many difficulties [1]. The social robot Probo will assist in providing information and moral support.

The development of the social robot Probo is part of the ANTY project, of which the main objective is to offer solutions to some specific needs of hospitalized children.

Another aspect of the ANTY project is the creation of a multi-disciplinary research community. The first prototype will be used as a test bed to investigate future possibilities and approaches to anticipate on arising social problems in Probo's work environment. Therefore, collaboration with pediatricians, sociologists and psychologists is a must. New opportunities, such as: Human-Robot Interaction (HRI) and Robot-Assisted Therapy (RAT), will be investigated.

\footnotetext{
* This work was supported by the Brussels-Capital Region.
} 
Besides the development of the prototype and the set up of a multi-disciplinary research community, the project also aims at being an educational stimulant for technological innovation by collaborations with other research groups and (high)schools.

This paper focuses on the conceptual ideas behind the robot Probo and some results of preliminary examinations that lead us to the actual prototype of an actuated robot head, capable of showing facial expressions.

\section{Concept Probo}

\subsection{Operational Goals}

In hospital, children need to be distracted from the scary and unfortunate hospital life e.g. by getting in contact with their family and friends. Furthermore, they require moral support and they have specific needs for relevant information about their illness, the hospital environment, medical investigations, etc. [1]. Several projects already exist that aim to use Information and Communication Technologies (ICT) like internet and webcams to allow hospitalized children to stay in contact with their parents, to virtually attend lectures at school and to provide information [2]. However, these ICT applications are usually computer animations displayed on PC, television screens or laptops. Moreover, people are used to interact with embodied creatures and have evolved communication skills, which both need a body for expression [3].

Recently Animal Assisted Therapy (AAT) and Animal Assisted Activities (AAA) are used in specific hospitals [4]. These kind of therapies and activities make use of animals to distract and to comfort the patients. AAT and AAA are expected to have useful psychological, physiological and social effects. However these animals are unpredictable and they can carry diseases. Very recently there were some experiments where robots were used in stead of animals for these kind of therapies. Using these social pet robots for therapeutic purposes has more advantages and a better chance of being allowed in the hospitals. For example, the seal robot Paro [5], [6], Sony's dog robot Aibo [7] and Philips' cat robot iCat [8] are being tested for Robot Assisted Therapy (RAT).

Bearing this in mind, the development of a 3D social robot, called Probo, has started. Communication will be the first focus of this robot. Probo is about $70 \mathrm{~cm}$ tall and equipped with a fully actuated head, an active vision system, an affective nonsense speech and a touch screen to comfort, inform and address children in a playful manner. The main goal for the robot Probo is to become a friend for the children.

\subsection{Huggable Imaginary Robot Animal}

Probo has to be seen as an imaginary animal based on the ancient mammoths. Its name is derived from the word Proboscidea, the order of animals with a proboscis, including the species of the elephant-like mammoths. The basic design of the robot is based on an imaginary animal, so that there is no exact similarity with well-known creatures. That way there are no or less expectations compared with a real animal. For instance, if a robot dog is presented, the children may be expected that the robot dog will bark like a real dog. 


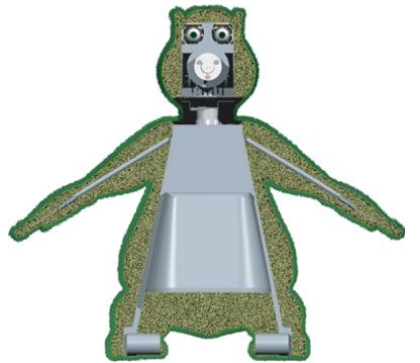

(a) Internal mechanics covered with soft materials, like foam.

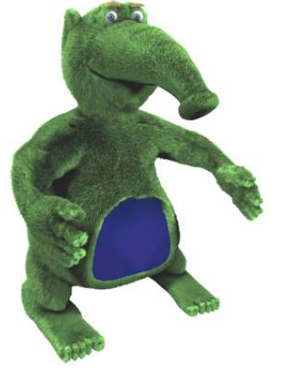

(b) Computerized 3D representation.



(c) Removable fur jacket.

Fig. 1: Concept of the huggable robot Probo.

Probo's huggable and soft appearance, intriguing trunk, and interactive belly-screen are striking. To communicate with the children, the robot is equipped with a fully actuated head, with actuated eyes, eyelids, eyebrows, ears, trunk, mouth and neck. The internal mechanics will be covered with soft and flexible materials, like foam, and on top of it a removable fur-jacket. Figure 1 shows a 3D computer model of the imaginary robot animal, Probo, and the way how the internal mechanics will be covered.

In [9], the relationship between color and emotion was tested, whereas the color green attained the highest number of positive responses (95.9\%), followed by the color

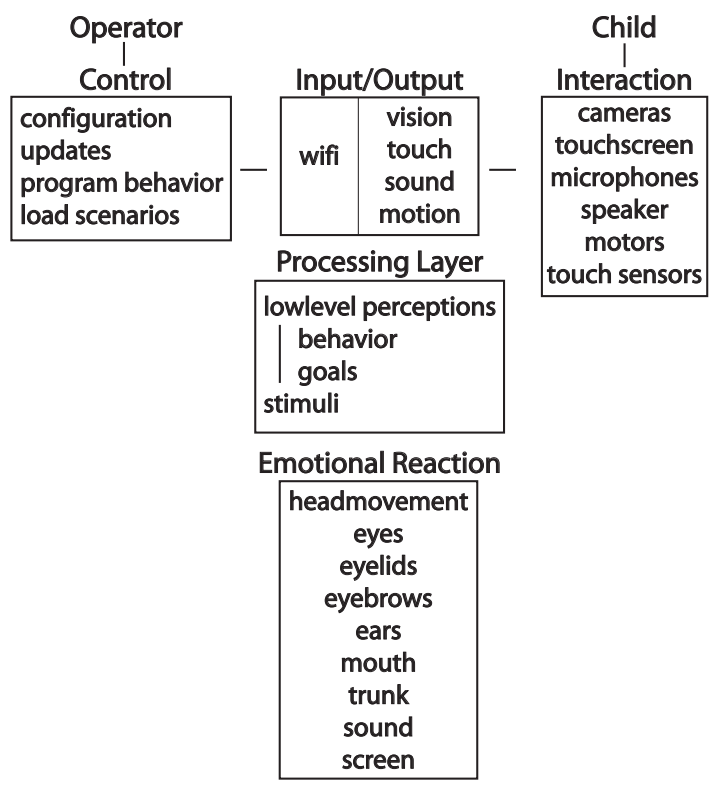

Fig. 2: Probo as a Robotic User Interface. 
yellow (93.9\%). The majority of emotional responses for the green color indicated the feelings of relaxation and calmness, followed by happiness, comfort, peace, hope, and excitement. The green color was associated with nature and trees, and thus creating feelings of comfort and soothing emotions. For these reasons Probo's color is chosen green.

\subsection{Multi-disciplinary Robotic User Interface}

Probo will function as a Robotic User Interface (RUI) between an operator and a child as shown in Figure 2. First, the robot will be controlled by an operator (caregivers, researchers, medical staff, etc.) who wants to communicate with the child. The robot functions as an interface that performs preprogrammed scenarios and reacts on basic input stimuli. The input stimuli, coming from low-level perceptions, are derived from vision analysis, audio analysis and touch analysis. Those stimuli will influence the attentionsystem and emotion-system, used to set the robot's point of attention, current mood and corresponding facial expression. The vision analysis includes the detection of faces, objects and facial features. Audio analysis includes detecting the direction and intensity of sounds and the recognition of emotions in speech.

A specific behavior-based framework is being developed to process these input stimuli. The framework is based on earlier work of Ortony, Norman and Revelle [10], who focus on the interplay of affect, motivation and cognition in controlling behavior. Each is considered at three levels of information processing: the reactive level is primarily hard-wired and has to assure the quick responses of the robot to make it look alive; the routine level provides unconscious, un-interpreted scenarios and automotive activity; and the reflective level supports higher-order cognitive functions, including behavioral structures and full-fledged emotions. Starting with a social interface, the reactive and routine level are being implemented. Currently, there is a shared control between the operator, configuring behavior, emotions and scenarios, and the robot, having basic autonomous reactions.

The development of such a social robot is very multi-disciplinary as shown in Figure 3. From the engineering point of view, the challenge is to build a safe and user friendly robot. For safe and soft interaction the joints need to be flexible, which can be obtained by incorporating compliant actuation. Compliant actuators are gaining interest in the robotic community. Pneumatic artificial muscles [11](such as McKibben muscles, Festo muscles, PPAM [12]), electric compliant actuators (such as VIA [13], AMASC [14] and MACCEPA [15]) and voice coil actuators [16] are some examples of compliant actuators. While some of them exhibit adaptable compliance, so that the stiffness of the actuated joint can be changed, it is not required in the Probo robot. Therefore, compliance is introduced by placing elastic elements between the motor and the actuated robot joint. In this way the external forces on the joint will be dissipated by the elastic elements.

The use of transferable mechanical and electronic components leads to an effective development and realization of the robot prototype. A modular mechanical and system architecture simplifies assemblage and maintenance. To realize a full-body sense of touch, a sensitive skin will be used. A good example is being developed (by Stiehl 
et al. [17]) for a therapeutic robotic companion named: The Huggable. In another approach, research has started for the use of photonic crystal fibers [18] which will be implemented in some parts of Probo, such as the trunk. Software for these sensors and for vision and speech analysis is developed as components. Component based software engineering emphasizes on decomposition of the engineered systems into functional or logical components with well defined interfaces used for communication across the components. Components are considered to be a higher level of abstraction than objects and as such they do not share state and communicate by exchanging messages carrying data.

\section{Actuated Robot head}

\subsection{Expression of Emotions}

In order to communicate and interact with humans following social rules, Probo needs the ability to express emotions. Therefore, a fully actuated head, for facial expressions, has been developed. In [19] the importance of facial expressions in human face-to-face communication is described. For the display of emotions most of the degrees of freedom (DOF) in its face are based on the Action Units (AU) defined by the Facial Action Coding System (FACS) developed by Ekman and Friesen [20]. AU express a motion of mimic muscles as 44 kinds of basic operation, with 14 well defined AU it becomes possible to express the emotions of anger, disgust, fear, joy, sorrow, and surprise. These emotions are often supported as being the 6 basic emotions from evolutionary, developmental, and cross-cultural studies [21]. Table 1 shows the different DOF compared with other actuated robot heads used for facial expressions. Figure 4a shows the prototype of the actuated robot head, with a total of 17 DOF [22]. Three additional DOF will enable the head to bend, nod and rotate.

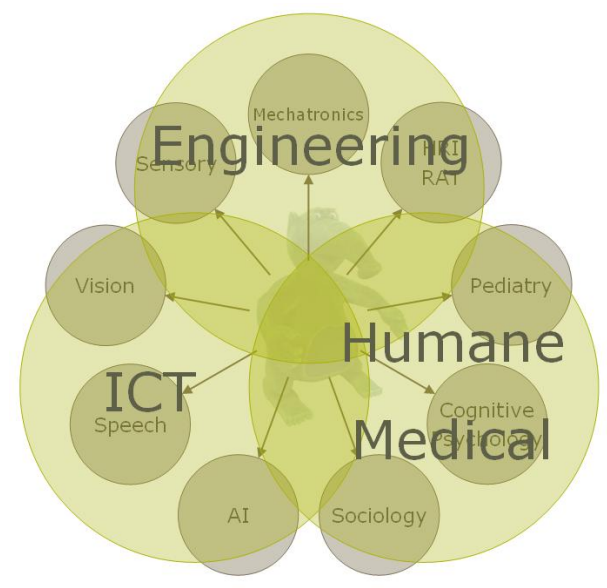

Fig. 3: Multi-disciplinary 
Table 1: DOF and ranges of the actuated joints of Probo's head in comparison with other non-humanoid robot heads.

\begin{tabular}{|c|c|c|c|c|c|}
\hline Kismet & Eddie & iCat & \multicolumn{3}{|c|}{ Probo } \\
\hline \multicolumn{4}{|c|}{ (DOF) } & \multicolumn{2}{|c|}{ Range $\left[{ }^{\circ}\right]$} \\
\hline Eyes (3) & Eyes (3) & Eyes (3) & Eyes (3) & $\begin{array}{l}\text { Pan } \\
\text { Tilt }\end{array}$ & \begin{tabular}{|c|}
100 \\
80
\end{tabular} \\
\hline Eyelids (2) & Eyelids (4) & Eyelids (2) & Eyelids (2) & & 150 \\
\hline Brows (4) & Brows (4) & Brows (2) & Brows (4) & & 45 \\
\hline Ears (4) & Ears (4) & & Ears (2) & & 90 \\
\hline Yaw (1) & Yaw (1) & & Mouth (3) & Yaw & 45 \\
\hline Lips (4) & Lips (4) & Lips (4) & & Lipcorners & \begin{tabular}{|l|l|}
560 \\
\end{tabular} \\
\hline Neck (3) & & Neck (2) & Neck (3) & $\begin{array}{l}\text { Rotate } \\
\text { Nod } \\
\text { Bend }\end{array}$ & \begin{tabular}{c|}
120 \\
70 \\
70
\end{tabular} \\
\hline & Crown (1) & & $\begin{array}{l}\text { Trunk (3) } \\
\end{array}$ & & 360 \\
\hline
\end{tabular}

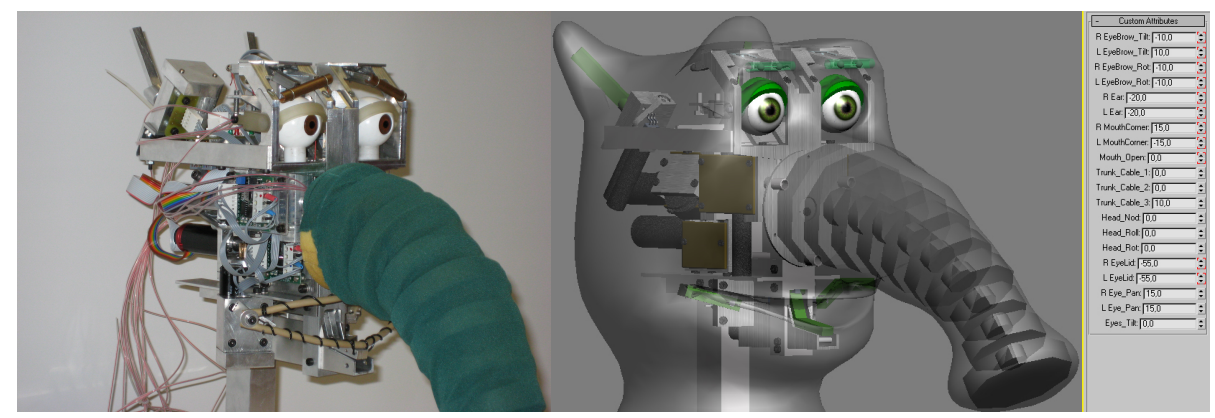

(a) Prototype of the robotic head.

(b) Virtual model of the robotic head.

Fig. 4: Probo's actuated head with 17 DOF: eyes (3 DOF), eyelids (2 DOF), eyebrows (4 DOF), ears (2 DOF), trunk (3 DOF) and mouth (3 DOF).

\subsection{Active Vision System}

Besides the role of the eyes to show some facial expressions, there are other reasons to equip a social robot with actuated eyes. The phenomenon that occurs when two people cross their gaze is called eye contact [23]. People use eye-gaze to determine what interests each other. The same phenomenon will be used between robot and child to encourage human robot interaction. By focussing the robot's gaze to a visual target, the person that interacts with the robot can use the robot's gaze as an indicator of its intentions. This facilitates the interpretation and readability of the robot's behavior, as the robot reacts specifically to what it is looking at [24]. This visual target will be referred to as the robot's point of attention (POA). Furthermore, when a robot is intended to interact with people, it requires an active vision system that can fulfill both a perceptual and a communicative function. An active vision system is able to interact with its 
environment by altering its viewpoint rather than passively observing it. Therefore, the designed eyes are hollow and contain small cameras. As these cameras can move, the range of the visual scene is not restricted to that of the static view.

The five DOF eyes module, used as active vision system in Probo, exists of two hollow eyeballs each mounted in an oribt as shown in Figure 5a. According to the chosen DOF based on the AU mentioned earlier; the eyes can pan separately and tilt together, each eye can be covered by an upper eyelid and the eyelids can blink separately. The eyebrows module fits on top of the eyes module. Each eyebrow has two DOF meaning that both the vertical position and the angle of each eyebrow can be set independently. Nine servomotors, together with a Bowden cable mechanism are used to power the eyes, eyelids and eyebrows. Axial springs and the usage of flexible cables both introduce compliance. Using flexible Bowden cables creates the opportunity to group and isolate the different servos and to place them anywhere in the robot. That way heat and noise dissipation can be controlled and the head can be held light-weighted, both resulting in a safe design.

\subsection{Attractive Trunk}

The trunk is a special part in contrast with most other robotic heads (eg. [3], [8], [25]) that use eyes, eyelids, eyebrows and a mouth for facial expressions. The proboscis or trunk of our robot appears to be the most intriguing element according to a small survey amongst children aged 10-13. It is used for grabbing and maintaining the child's attention. When the child's attention is focused on the trunk, the child's face fits within the range of the on board eye cameras. This simplifies the recognition of children's mood or emotional status. In this way the robot can react properly to different situations and it intensifies certain emotional expressions and it increases interactivity.

The three DOF trunk, as shown in Figure 5b, consists of a foam core with segmented extension discs. Axial to the centerline, three flexible cables are guided through the discs and attached to the front disc. The end of each cable is attached to a wind-up

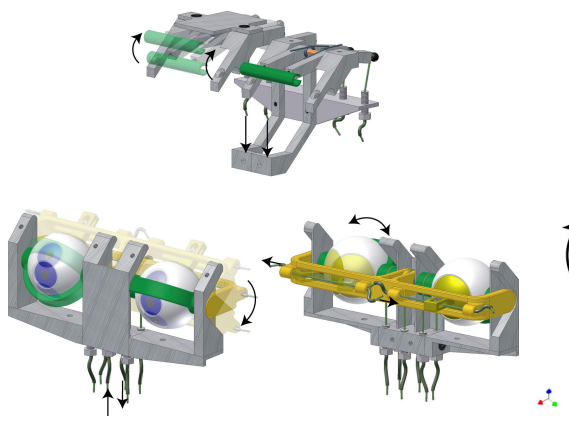

(a) CAD model of the Eyes and Eyebrows.

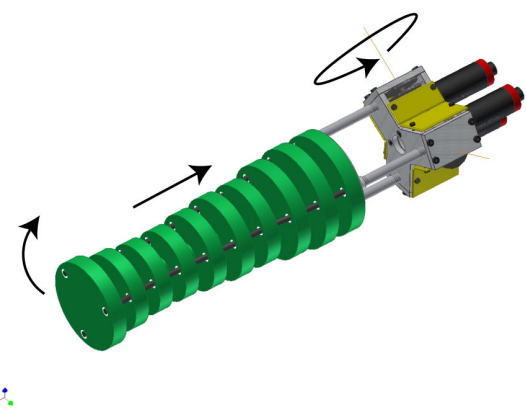

(b) CAD model of the Trunk.

Fig. 5: Probo's eyes and trunk. 
pulley resulting in a motion of the entire trunk. The motion of the trunk depends on; the number of discs, the dimensions of the discs and the core, the flexibility of the cables and the composition of the foam. A high compliance and durability of the trunk is ensured by using a foam material actuated by flexible cables. Interaction with this trunk will be safe both for the child, that can not get hurt, and for the motors, that can not be broken.

\subsection{Recognition Tests}

A virtual model (Figure 4b) of Probo has been created to evaluate the design. The model combines the mechanical designs with the visual exterior of the robot, represented by the skin, attached to the mechanical moving parts. The mechanical parts are linked together to obtain kinematical movements for realistic visual motions of the model. The movements can be controlled with sliders to set the desired angles for the DOF and simulating actuation of the parts. To test the recognition of facial expression, the virtual model was used in a preliminary user-study. The study was based on a survey performed by Cynthia Breazeal evaluating the expressive behavior of Kismet [3]. We asked the subjects to compare renderings of the virtual model (Figure 6b) with a series of line drawings of human expressions (Figure 6a).

Twenty-five subjects ( 6 - 8 years of age) filled out the questionnaire. The children were presented an image of our virtual model representing one of the 8 emotions. For each of those images they had to choose the best matching sketch representing human emotions. The results are shown in Table 2. The results from the test show that the intended emotions surprise, fear and happy have a low similarity with the sketches. Because the sketches contain also a drawing stating a pleased emotion, the low result for happy can be explained. Combing the two gives even a $90 \%$ similarity between the happy emotion and a happy or pleased human face. The image expressing fear was often related to sorrow and pleased. There is a strong resemblance between the images representing fear and sorrow (15\%). This can partly be explained because our model lacks lower eyelids resulting in a smaller difference in eye-opening. The lowest similarity was found with the surprise emotion, where slightly more children linked the surprise image with the fear sketch (29\%). During the test, the observation was made

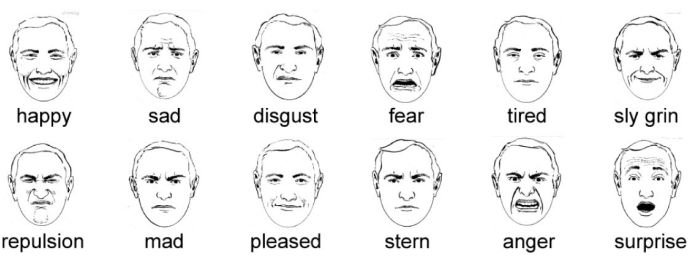

(a) The sketches used in the evaluation, copied from Kismets survey, adapted from (Faigin 1990) [3].

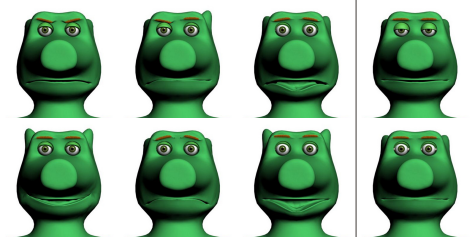

(b) The 6 basic emotions (anger, disgust, fear, happy, sad and surprise) on the left and the expressions tired and neutral on the right.

Fig. 6: Facial expressions used in preliminary user-study. 
Table 2: The result of the comparison test with children shown in percentage match.

\begin{tabular}{|c|c|c|c|c|c|c|c|c|}
\hline$\%$ match & happy & sad & disgust & mad & fear & tired & surprise & neutral \\
\hline happy & $\mathbf{5 4}$ & 0 & 7 & 0 & 0 & 0 & 18 & 0 \\
\hline sad & 0 & $\mathbf{7 4}$ & 9 & 7 & 15 & 2 & 0 & 0 \\
\hline disgust & 0 & 4 & $\mathbf{6 2}$ & 4 & 3 & 0 & 0 & 4 \\
\hline mad & 1 & 2 & 2 & $\mathbf{6 6}$ & 3 & 9 & 0 & 16 \\
\hline fear & 0 & 0 & 0 & 0 & $\mathbf{4 8}$ & 0 & 29 & 0 \\
\hline tired & 0 & 4 & 5 & 2 & 0 & $\mathbf{8 7}$ & 3 & 4 \\
\hline surprise & 0 & 0 & 0 & 0 & 9 & 0 & $\mathbf{2 8}$ & 0 \\
\hline sly grin & 5 & 0 & 2 & 11 & 5 & 0 & 0 & 0 \\
\hline stern & 0 & 12 & 9 & 0 & 2 & 0 & 0 & 40 \\
\hline anger & 2 & 0 & 0 & 3 & 0 & 0 & 7 & 4 \\
\hline repulsion & 2 & 4 & 0 & 7 & 3 & 0 & 0 & 0 \\
\hline pleased & 36 & 0 & 4 & 0 & 12 & 2 & 15 & 32 \\
\hline
\end{tabular}

that the children were really seeking for a visual resemblance without recognizing the underlying emotions.

When performing the same test on fifteen adult people (20 - 35 years of age) the results in Table 3 were similar with the exception of surprise. Where the children had difficulties identifying the emotion of surprise most of the adults $(81 \%)$ had a positive match. We also observed that some of the adults, first try to recognize the underlying emotions rather than just look for a graphical similarity, resulting in better matches.

Table 3: The result of the comparison test with adults shown in percentage match.

\begin{tabular}{|c|c|c|c|c|c|c|c|c|}
\hline$\%$ match & happy & sad & disgust & mad & fear & tired & surprise & neutral \\
\hline happy & $\mathbf{5 6}$ & 0 & 0 & 0 & 6 & 0 & 13 & 0 \\
\hline sad & 0 & $\mathbf{8 8}$ & 0 & 0 & 44 & 13 & 0 & 6 \\
\hline disgust & 0 & 6 & $\mathbf{6 3}$ & 0 & 0 & 0 & 0 & 0 \\
\hline mad & 0 & 0 & 6 & $\mathbf{6 9}$ & 0 & 0 & 0 & 6 \\
\hline fear & 0 & 0 & 0 & 0 & $\mathbf{4 4}$ & 0 & 0 & 6 \\
\hline tired & 0 & 0 & 6 & 6 & 0 & $\mathbf{8 1}$ & 0 & 44 \\
\hline surprise & 0 & 0 & 0 & 0 & 0 & 0 & $\mathbf{8 1}$ & 6 \\
\hline sly grin & 19 & 0 & 6 & 0 & 0 & 0 & 0 & 0 \\
\hline stern & 0 & 6 & 19 & 19 & 6 & 0 & 0 & 19 \\
\hline anger & 0 & 0 & 0 & 6 & 0 & 0 & 0 & 0 \\
\hline repulsion & 0 & 0 & 0 & 0 & 0 & 0 & 0 & 0 \\
\hline pleased & 25 & 0 & 0 & 0 & 0 & 6 & 6 & 13 \\
\hline
\end{tabular}




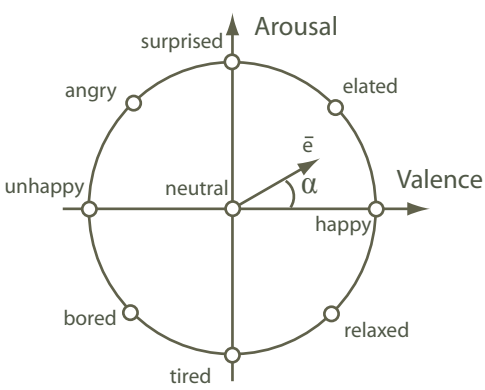

(a) Emotional space based on Russells circomplex model of affect.

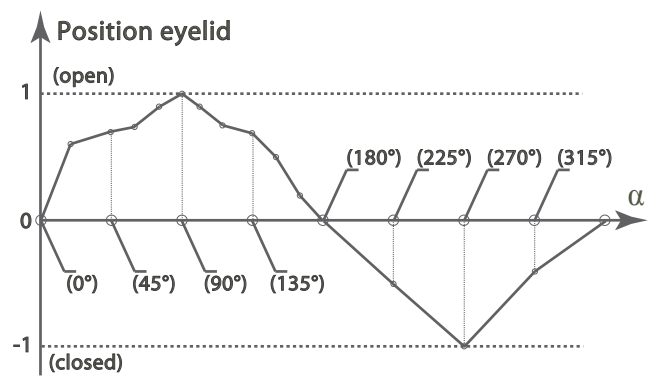

(b) Adjustable interface for defining the values of the DOF, in this case an eyelid, for each emotion.

Fig. 7: Display of emotions.

\section{Emotional Interface}

To realize a translation from emotions into facial expressions, emotions need to be parameterized. In [3], Kismet's facial expressions are generated using an interpolation based technique over a three-dimensional, componential affect space (arousal, valence, and stance). Cf. [3] our model has two dimensions; valence and arousal to construct an emotion space, based on the circumplex model of affect defined by Russell [26].

Figure 7a shows the emotion space of Probo. The x-coordinate represents the valence and the y-coordinate the arousal, consequently each emotion $e(v, a)$ corresponds to a point in the valence-arousal-plane (Figure 7a). In this way we can specify basic emotions on a unit circle, placing the neutral emotion $e(0,0)$ in the origin of the coordinate system. Now each emotion can also be represented as a vector with the origin of the coordinate system as initial point and the corresponding arousal-valence values as the terminal point. The direction $\alpha$ of each vector defines the specific emotion whereas the magnitude defines the intensity of the emotion. The intensity $i$ can vary from 0 to 1 , interpolating the existing emotion $i=1$ with the neutral emotion $i=0$. Each DOF that influences the facial expression is related to the current angle $\alpha$ of the emotion vector. An adjustable interface is developed to define the desired value for each angle $\left(0^{\circ}-360^{\circ}\right)$ of the different DOF. By selecting one degree of freedom, we set a value for each basic emotion on the unit circle and use linear interpolation to obtain a contiguous relation. By adding more (optional) points or values the curve can be tuned to achieve smooth, natural transitions between different emotions. An example for the degree of freedom controlling the eyelid is shown in Figure $7 \mathrm{~b}$.

A graphical user interface (GUI) (Figure 8) has been developed wherein the user can fully configure the facial expressions and use the emotion space to test the different emotions and transitions. The user will obtain visual feedback from a virtual model of the robot. In addition to the facial expression this interface has been extended with a component controlling the point of attention. This component controls the eyes and neck motion according to a specific point in the three dimensional space. The respective coordinates of that point can be altered in real time and will be represented as a 


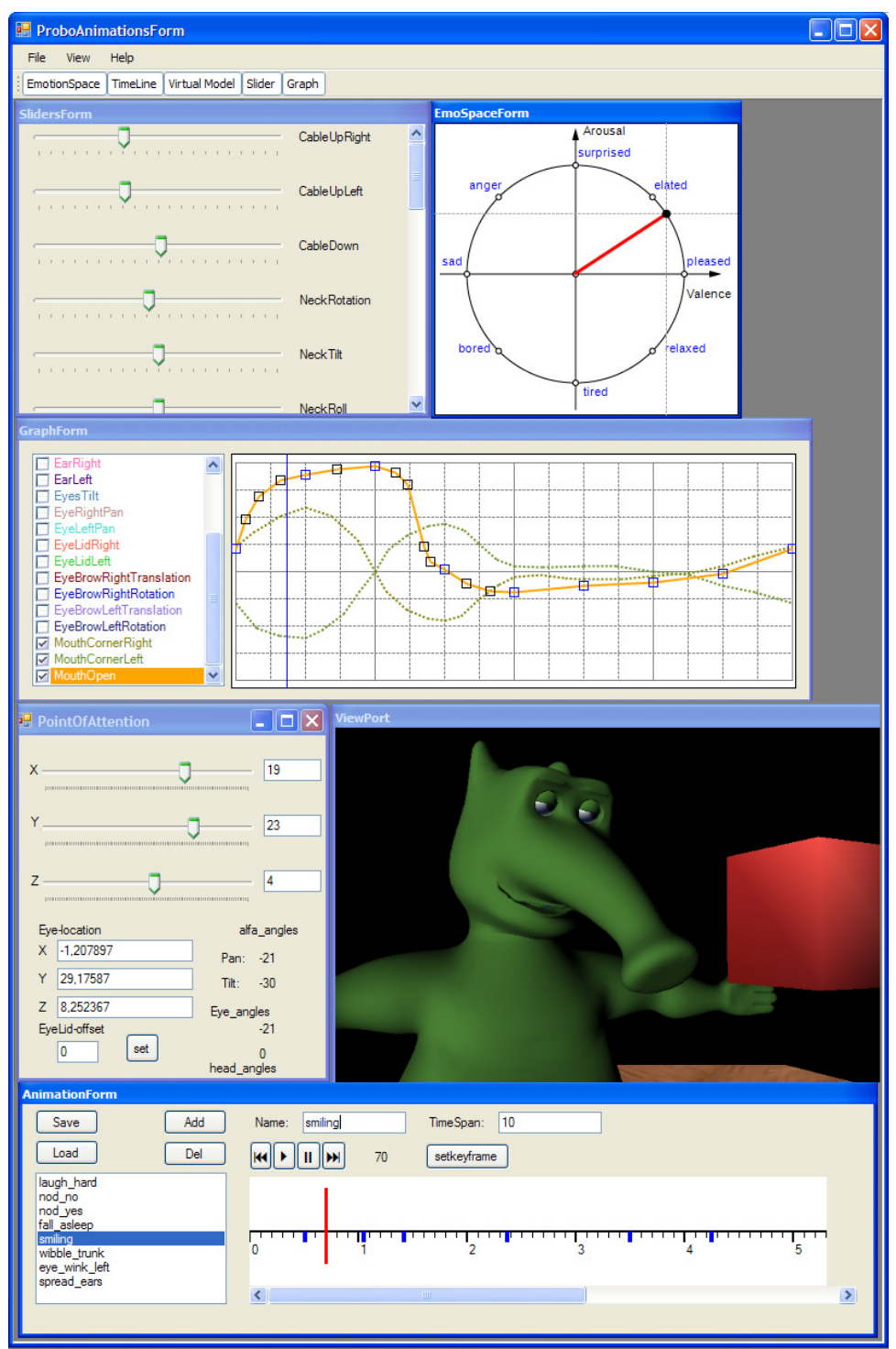

Fig. 8: Graphical User Interface. 
red cube in the virtual space. This coordinate is translated into rotation angles for the 4 DOF controlling the eyes (pan/tilt) and the head (pan/tilt). As part from the vision analysis, a face recognition component is developed using Intel $\mathbb{R}$ 's OpenCV library. This component uses a webcam to capture the images and then calculates the center of the face as a cartesian coordinate. This coordinate can then be used to control the point of attention in the virtual space. Another component in this interface gives the user the ability to create animations, store, edit and play them. Each animation consists of different key frames, which hold the values of the DOF at a given time. There is a linear interpolation between the different key frames resulting in a contiguous animation. The emotional interface can be used to easily insert emotions at a certain point in an animation. The different animations are stored in a database and will be employed later to build scenarios for the robot.

\section{Conclusion}

To inform and comfort hospitalized children in a playful way, the development of a new social robot, Probo is started. Probo's main goal is to communicate with the children. Therefor a fully actuated robot head, capable of showing facial expressions, is designed. The degrees of freedom in the head are based on the action units defined in the facial action coding system. Compared with other non-humanoid robot heads Probo has an intriguing trunk. By us of soft and flexible materials together with compliant actuators, the design is safe and as a consequence safe human robot interaction is assured. With this multidisciplinary test-bed, new opportunities like robot assisted therapy will be explored in collaboration with pediatricians, sociologists and psychologists. To control the robot, an emotional graphical user interface has been developed. With this interface an operator can set all degrees of freedom of the virtual and at the same time linked real model. Different emotions can be showed by moving a cursor in a $2 \mathrm{D}$ emotion space.

\section{References}

1. Vanderfaeillie, J., Y., V.: Zieke kinderen en jongeren. In: Handboek orthopedagogische hulpverlening. Deel 1. Een orthopedagogisch perspectief op kinderen en jongeren met problemen. Leuven/Voorburg: Acco (2005) 199-237

2. Fels, D., Shrimpton, B., Roberston, M.: Kids in hospital, kids in school. In Lassner, D., McNaught, C., eds.: Proceedings of World Conference on Educational Multimedia, Hypermedia and Telecommunications 2003, Honolulu, Hawaii, USA, AACE (2003) 2358-2363

3. Breazeal, C.: Designing Sociable Robots. Mit Pr (2002)

4. Burch, M.: Animal-assisted therapy and crack babies: A new frontier. Pet Partners Program: A Delta Society Newsletter (1991)

5. Shibata, T., Mitsui, T., Wada, K., Touda, A., Kumasaka, T., Tagami, K., Tanie, K.: Mental commit robot and its application to therapy of children. Advanced Intelligent Mechatronics, 2001. Proceedings. 2001 IEEE/ASME International Conference on 2 (2001)

6. Shibata, T., Wada, K., Saito, T., Tanie, K.: Robot Assisted Activity for Senior People at Day Service Center. Proc. of Intl Conf. on Information Technology in Mechatronics (2001) 71-76 
7. Tamura, T., Yonemitsu, S., Itoh, A., Oikawa, D., Kawakami, A., Higashi, Y., Fujimooto, T., Nakajima, K.: Is an Entertainment Robot Useful in the Care of Elderly People With Severe Dementia? Journals of Gerontology Series A: Biological and Medical Sciences 59(1) (2004) 83-85

8. van Breemen, A.: iCat: Experimenting with Animabotics. Proceedings, AISB 2005 Creative Robotics Symposium (2005)

9. Kaya, N., Epps, H.: Relationship between Color and Emotion: A Study of College Students. College Student Journal 38(3) (2004) 396-406

10. Ortony, A., Norman, D., Revelle, W.: 7 Affect and Proto-Affect in Effective Functioning. Who Needs Emotions?: The Brain Meets the Robot (2005)

11. Daerden, F., Lefeber, D.: Pneumatic artificial muscles: actuators for robotics and automation. European Journal of Mechanical and Environmental Engineering 47(1) (2002) 11-21

12. Verrelst, B., Van Ham, R., Vanderborght, B., Lefeber, D., Daerden, F., Van Damme, M.: Second generation pleated pneumatic artificial muscle and its robotic applications. Advanced Robotics 20(7) (2006) 783-805

13. Tonietti, G., Schiavi, R., Bicchi, A.: Design and control of a variable stiffness actuator for safe and fast physical human/robot interaction. Proceedings of the IEEE International Conference on Robotics and Automation (2000) 528-533

14. Hurst, J., Chestnutt, J., Rizzi, A.: An actuator with physically variable stiffness for highly dynamic legged locomotion. Robotics and Automation, 2004. Proceedings. ICRA'04. 2004 IEEE International Conference on $\mathbf{5}$

15. Van Ham, R., Vanderborght, B., Van Damme, M., Verrelst, B., Lefeber, D.: MACCEPA, the mechanically adjustable compliance and controllable equilibrium position actuator: Design and implementation in a biped robot. Robotics and Autonomous Systems 55(10) (2007) 761-768

16. McBean, J., Breazeal, C.: Voice Coil Actuators for Human-Robot Interaction. IEEE. RSJ International Conference on Intelligent Robots and Systems (IROS04), Sendai, Japan (2004)

17. Stiehl, W., Lieberman, J., Breazeal, C., Basel, L., Lalla, L., Wolf, M.: Design of a therapeutic robotic companion for relational, affective touch. Robot and Human Interactive Communication, 2005. ROMAN 2005. IEEE International Workshop on (2005) 408-415

18. Urbanczyk, W., Martynkien, T., Szpulak, M., Statkiewicz, G., Olszewski, J., Golojuch, G., Wojcik, J., Mergo, P., Makara, M., Nasilowski, T., et al.: Photonic crystal fibers: new opportunities for sensing. Proceedings of SPIE 6619 (2007) 66190G

19. Mehrabian, A.: Communication without words. Psychology Today 2(4) (1968) 53-56

20. Ekman, P., Friesen, W.: Facial Action Coding System. Consulting Psychologists Press (1978)

21. Ekman, P.: Are there basic emotions? Psychological review 99(3) (1992) 550-553

22. Goris, K., Saldien, J., Vanderborght, B., Lefeber, D.: The Huggable Robot Probo: Design of the Robotic Head. AISB symposium (accepted) (2008)

23. Breazeal, C.: Regulation and Entrainment in Human-Robot Interaction. Experimental Robotics VII (2001)

24. Miyauchi, D., Sakurai, A., Nakamura, A., Kuno, Y.: Human-robot eye contact through observations and actions. Proceedings of the 17th International Conference on Pattern Recognition, 2004. ICPR 2004. 4 (2004)

25. Beira, R., Lopes, M., Praça, M., Santos-Victor, J., Bernardino, A., Metta, G., Becchi, F., Saltaren, R.: Design of the Robot-Cub (iCub) Head. Proc. IEEE International Conference on Robotics and Automation, ICRA, Orlando, May (2006)

26. Posner, J., Russell, J., Peterson, B.: The circumplex model of affect: An integrative approach to affective neuroscience, cognitive development, and psychopathology. Development and Psychopathology 17(03) (2005) 715-734 